\title{
Italique
}

Poésie italienne de la Renaissance

XIX | 2016

Varia

\section{Preliminari a uno studio delle lettere di Giovanni} Battista Leoni

\section{Roberto Gigliucci}

\section{(2) OpenEdition}

Journals

\section{Edizione digitale}

URL: http://journals.openedition.org/italique/435

DOI: $10.4000 /$ italique.435

ISSN: 1663-4438

\section{Editore}

Librairie Droz

\section{Edizione cartacea}

Data di pubblicazione: 1 dicembre 2016

Paginazione: 167-188

ISBN: 978-2-600-04777-7

ISSN: 1423-3983

Notizia bibliografica digitale

Roberto Gigliucci, « Preliminari a uno studio delle lettere di Giovanni Battista Leoni », Italique [Online], XIX | 2016, online dal 01 décembre 2018, consultato il 07 septembre 2019. URL : http:// journals.openedition.org/italique/435; DOI : 10.4000/italique.435 


$$
\text { R овеR Tо GIGLIUCC I }
$$

\section{P RELIMIN A R A U N O ST U D I O}

D E L L E L E T T E R E

DI GIOVANNI BATTITA LEONI 



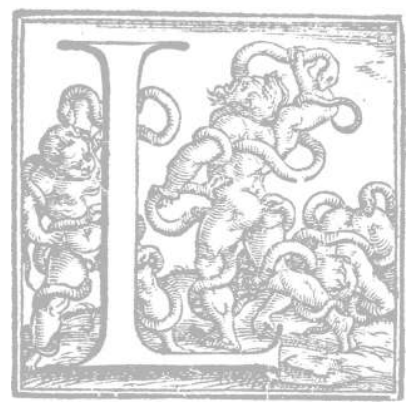

'attività letteraria di Giovanni Battista Leoni fu intensa, poliedrica e sperimentale; un caso, si sarebbe detto in altri tempi, di poligrafia. ${ }^{\mathrm{I}}$ Autore teatrale assai ambizioso e riccamente propositivo, scrisse una «tragisatiricomedia», la Roselmina, che firmò con lo pseudonimo di Lauro Settizonio da Castel Sambucco, ${ }^{2}$ testo che si meriterà pure una citazione dal grande Lope de Vega. Questi infatti, nel prologo-dedica della «comedia famosa» Las Almenas del Toro $^{3}$ si esprime così, difendendo la natura mezclada dell'innato tragicomico ispanico e pur trovandone almeno una traccia arricchita in suolo italico:

O gran fuerça de la novedad, pues ya en Italia, a esta mezcla de estilos, se añadio otro, ${ }^{4}$ si bien en la comedia estuvo siempre incluso, pues en su lengua anda una impressa con este titulo: Tragisatyricomedia. ${ }^{5}$

\section{Nel prologo della Roselmina, l'opera è definita}

capricciosissima; è un composito di faceto, \& di serio; di grave, \& di giocoso; un mescuglio di Prencipi, \& di gente bassa, e mezana, allegra, desperata, pazza, e savia; un intrecciamento di negotij grandi, \& di burle giocondissime; con discorsi, \& pensieri di Donne, Cavalier, d'armi, \& d'amori; accomodati in modo, che nella loro discorde convenienza, fanno una gentilissima, et harmonica compositione. [p. 6 della princeps]

Sembrerebbe un prodotto di grande sintesi di tutti gli elementi discordanti che in quei tempi si andavano sempre più raggrumando in forme teatrali polimorfe e tragicomiche. In realtà la commedia del Leoni non presenta situazioni e personaggi che non siano piuttosto collaudati, pur se l'ibridismo amato dal gusto del pubblico coevo è alquanto rispettato. Anche la Florinda del i607 (Venezia, Ciotti, firmata col medesimo pseudonimo della Roselmina) si presenta come un prodotto teatrale insolito; il frontespizio recita Florinda Grottesca Dramatica, overo Favola Eteroclita, e nella dedica a Girolamo Lanza l'autore rammenta $i$ «capricci» del proprio «cervellaccio»; il Gobbo che recita il prologo si produce poi in una parallela lode paradossale della difformità propria e della pièce, anormalità utile e dilettevole (si pensi al 
contrasto con il monologo iniziale del Richard III!). Il Leoni pubblica altresi una «tragicommedia spirituale», La conversione del peccatore a Dio (Venezia, Francesco de' Franceschi, I59I, I592, poi ivi, Pietro Farri, I6I3), in cui rifutando sia il modello comico classico, sia quello tragico altrettanto classico, si produce in un ibrido morale, o meglio in un pezzo di teatro spirituale allegorico (con personaggiemblemi quali l'Uomo, la Natura, il Mondo, l'Intelletto, il Peccato ecc., con nomi grecizzati) di forte motivazione controriformistica, un genere su cui poco si è fatta luce, che sarà più tardi rappresentato ad esempio dal Parthenio di Francesco Pona, ${ }^{6}$ e che proprio nel I60o a Roma trova una congiunzione col neonascente «stile recitativo» musicale nella Rappresentazione di anima e corpo di Manni-Cavalieri, in ambiente filippino. Altra favola allegorica, faceta e insieme honesta, veritiera, insomma "grave», è La falsa riputatione della Fortuna, che fu urecitata dagli Academici Generosi del Seminario Patriarcale di Venetia», come si legge nel frontespizio (Venezia, G. B. Ciotti, I596, 1598 ecc.): anche qui intorno a un "Neandro cioè Huomo» si muovono personaggi allegorici dal nome ellenizzato, in una noiosa vicenda temperatamente anticortigiana ove la Virtù e soprattutto la Pazienza sconfiggono la Fortuna. "Tragicomedia», nella forma elevata di una tragedia in versi a scioglimento lieto, ${ }^{7}$ è invece l'Antiloco (Ferrara, Benedetto Mammarelli ad istanza di G. B. Ciotti, I594), dedicato a Giovanni de' Medici, mentre alla granduchessa Cristina di Lorena era dedicata La conversione e a Vincenzo Lodovici la Riputatione, cioè allo stesso che curerà le didascalie nella riproposta I598 dei Madrigali, su cui torneremo. ${ }^{8}$

Certo il Leoni è noto soprattutto per le Considerationi sopra l'Historia d'Italia di messer Francesco Guicciardini (Venezia, Giolito, I583 e altre edizioni seguenti), in cui accusava lo storico di parzialità anti-veneziana e prendeva le difese della propria patria. Altra opera di ambito storico, legata al periodo di servizio del Leoni presso il Duca di Urbino (I598-1602)9 ma dedicata «alla Sereniss.ma Republica di Venetia», è la Vita di Francesco Maria di Montefeltro della Rovere, per il solito Ciotti (Venezia I605), su cui peraltro il Guarini fece ricche annotazioni, conservate dal ms. 1313 dell'Angelica di Roma. ${ }^{\text {IO }}$ Non poche poi le orazioni, encomiastiche, funebri, sacre, che il Leoni diede alle stampe, e non si dimentichino $i$ Due discorsi sopra la libertà ecclesiastica di Giovan Simone Sardi Venetiano 
(Anno M.D.CVI, senza altre note tipografiche), ${ }^{\mathrm{II}}$ che sono in effetti un documento scottante relativo alla vicenda dell'Interdetto contro Venezi ia: vi si sostiene che il Papa non è Dio, che il potere ecclesiastico non pù̀ conculcare quello dei Principi (tale per ius divinum), che la chiesa non può estendere il suo potere temporale sull'universo, intrudendosi negli Stati e sovvertendoli; infine si prende la difesa della Repubblica contro l'ingerenza della Curia. Tutto questo, da parte di un intellettuale che aveva servito prima il cardinal Commendone, poi il card. Luigi d'Este, quindi il card. Lenoncourt, insomma era vissuto nelle segreterie ecclesiastiche per più di due decenni. Al suo discepolo Lodovici che vuole farsi segretario, da Rovigo nel Io lug. '95, scrive di aver avuto pratica di segreteria da ben 24 anni (Delle lettere del Signor Gio. Battista Leoni. Parte terza. Nella quale si contengono negotij, avvisi, et complimenti gravissimi. Raccolte et publicate da Vincenzo Lodovici. [...] In Venetia, M.D.XCVI. Appresso Gio. Battista Ciotti Senese, al segno della Minerva, cc. 26v-27r). Ma ancora lavorerà, come abbiamo accennato sopra, presso il duca d'Urbino Francesco Maria II, del cui nonno verga la voluminosa biografia, e l'ambiente urbinate è testimoniato da più d'una rubrica del poco letto volume De' madrigali e rime di Giovan Battista Leoni. Parte terza. [...] In Venetia, M.DCIV, Appresso Gio. Battista Ciotti Senese All'Aurora, dedicato al Conte Giulio Cesare Mamiani della Rovere. Ivi i madrigali sono 48, l'ultimo è una «Mascherata di Pescatori»; segue una «Mascherata e ballo di Nereide fatto in Pesaro il primo anno delle nozze della Serenissima Signora Donna Livia della Rovere Duchessa d'Urbino», in strofe esastiche di endecasillabi e settenari; conclude un'"Oda per l'acque del parco di Castel Durante ridotte in fonte e peschieras, sempre in strofette esastiche di endecasillabi e settenari, avendo così in totale so pezzi, come già nella serie prima e nella seconda. Su questa terza serie di madrigali, della cui stampa conosco al momento l'esemplare conservato presso la biblioteca Casanatense di Roma, ${ }^{\mathrm{I} 2}$ abbiamo già detto qualcosa altrove. ${ }^{\mathrm{I}}$

Leoni si segnala come rimarchevole epistolografo: raccolse infatti non poche sue lettere, di cui talune piene di gusto e umore, in una prima serie (Delle lettere familiari [...] Parte prima, Venezia, G. B. Ciotti, I592, rist. I593), in una seconda (ivi, I593) e infine in una terza (ivi, I596), come già detto curata dal Lodovici. Cercheremo, in questi primi esitanti passi nell'analisi di un corpus di lettere così interessante per 
molti versi, di isolare alcuni luoghi più aderenti alle tematiche letterarie, poetiche e teatrali che hanno coinvolto il nostro.

Per cominciare trascriviamo una missiva dalla Parte prima (citando dalla ristampa del I593, cc. 32r-v), in cui è lampante la modalità della nascita di un componimento poetico d'occasione, genere nel quale il madrigalista Leoni eccelleva:

A Monsignor Fabio Orsini, ${ }^{14}$ Roma.

Standomene ultimamente così solo considerando i corsi varij, gli aspetti, le illuminationi, gli ecclipsi, le conversioni, i satellitij i concorsi, gli orti, \& gli occasi; \& in fine misurando \& osservando metodicamente i viaggi; \& le circonvolutioni tutte delle stelle fisse \& erranti del mio globo rognoso; argomentando con qualche Zoroastrica ragione quali influssi, \& quali inclinationi potessero cagionare così fatte costellationi ne i corpiccini colerici, \& saltanti; \& altri di diversa complessione, che si generano tra le lenzuola sotto la loro virtù \& calore. Et mentre appunto che m'ero fermato sopra certo aggregato di segni, che mi rappresentavano al naturale la chioma di Berenice, \& che andavo rissolvendo di volerne calcular \& estender le efemeride, per dedicarle poi a V. S. Illustriss. ne i futuri Comitij Numentani; mi sovvenne di quello, che le piacque già di commandarmi narrandomi quel caso amoroso di quella Dama, ch'essendosi lavata la testa, \& havendo stese et esposte le belle chiome al sole, per favorir l'amante che passava in cocchio sollevò un tantino i penduli \& ruggiadosi crini, che $<$ cortesemente $>$ da così fatto moto stillarono certo poco liquore. Onde che portato egualmente dal voler servire a quel Cavaliero, et da non so che di furore incognito, me ne passai dalla speculatione rognosa alla prattica versificatoria. M'è riuscito però non so come questa massa di alcuni pochi versacci, che io non saprei veramente come intitolarla: \& se ciò non havesse a servirmi per attestar a V. S. Illustrissima la riverente servitù, con la quale vive in me l'obligo, che le devo, sicuramente, che non glielo lasciarei vedere. Consentisi adunque d'accettar la compositione qual ella si sia; \& l'affetto del mio cuore con quella benignità che suole. \& le bacio le mani.

Di Parigi a 4. di Giugno I $587 .^{\text {Is }}$

Da nube d'oro intorno

Adombrato il mio sole, mi facea

Notte provar nel più sereno giorno;

Ond'io qual pellegrino, 
Cui le tenebre rompino il camino,

Rivolgo gli occhi al ciel per farmi almeno

Del fosco lume suo guida fra via.

Et ecco fiammeggiar caro baleno

Di man pietosa, che la nube aperse,

$\mathrm{E}$ in lieta pioggia il dì chiaro m'offerse.

Quindi i rai del mio sol nascosti pria

Col reflesso di queste

Stille felici nella fronte altera

Spiegar ratti un leggiadro arco celeste.

$\mathrm{Ah}$, che più dunque errar di sfera in sfera?

Ecco al moto, \& al lume, \& al calore,

Madonna Sol del Mondo, \& Ciel d'Amore.

Si tratta di una delle tante situazioni liriche tardo-cinquecentesche e poi barocche (con le dovute evoluzioni di stile e concettismo) per le quali l'assenza di una rubrica esplicitante rende quasi impossibile l'interpretazione. Tuttavia la lettera aggiunge ancora qualcosa di più, uno shift giocoso: "me ne passai dalla speculatione rognosa alla prattica versificatoria». Ovvero dal delirante cosmo astrale delle pulci nel letto del nostro, alla scrittura di versi intorno a un'occasione del tutto prosaica (la Dama si leva $i$ capelli bagnati dal viso), la cui asemanticità è però immediatamente caricata di senso e bellezza perché riguarda, appunto, la donna amata. Ecco che allora possiamo produrre una prima riflessione: il madrigale-occasione è componimento con una sua autonomia (e un suo bisogno di rubrica esplicativa), ma inserito nell'ambito di una lettera burlesca sembra trovare un contesto più ampio, blandamente narrativo ma riccamente concettistico, sulla strada dell'iperbole continuata. La storia della lirica di ogni tempo andrebbe sempre integrata con le eventuali situazioni epistolografiche in cui $i$ componimenti si trovino incastonati.

Inoltre c'è da dire che il motivo basso-comico di pulci e pidocchi, già cinquecentesco, ba uno sviluppo incredibile nel Seicento; senza voler pensare a vertici metafisico-amorosi come The Flea di John Donne, pensiamo agli spidocchiamenti vari dell'iconografia pittorica, da un De La Tour a un Crespi. Citiamo però almeno la graziosa storiella della pulce e del pidocchio che si spartiscono le «morbide membra» e sanguigne di una fanciulla nel suo «morbido letto», procurandole con i loro morsi «certe rose, che se un dipintore havesse voluto ritrarre una primavera, 
intrecciata con una nevosa vernata, non haverebbe presa altra sembianza», il tutto raccontato da Agnolo Firenzuola. ${ }^{16}$

Sul motivo comico-basso degli insetti molesti Leoni ritorna in una saporosa lettera del secondo volume di epistole. ${ }^{17}$ Scrive al nobile Pietro Badoaro, che è a Venezia, da Firenze, il 29 agosto '8I. Lo stile realista e insieme concettista si squaderna in questo tipo di missive costruite proprio per intrattenere e divertire il destinatario. Siamo esattamente sul discrimine tardo-cinquecentesco che lega la memoria aretiniana alla prosa rigogliosa del Seicento.

Sono in camicia, e sbraccato per difendermi dal fastidio del caldo, ma sono così tormentato dalle mosche e da i pulci, che moverei riso e compassione alle statue. Convengo scrivendo far mille pause, mille salti, e torcimenti di vita, \& incrudelire bene spesso con me medesimo percuotendo indiscretamente hora questa hora quell'altra parte del corpo, sperando pure di coglier qualcuno di que' diabolici animali. Tra i quali una prosontuosa, et insolente quadriglia di mosche, risolute di voler farmi una moresca sulla punta del naso, mi ha havuto a far impazzare: l'ho disturbate più volte con la penna: con le mani due volte mi sono dato così fieri colpi, che ò havuto a snasarmi: \& finalmente ritornando pure le manigolde alla tresca principiata mi condussero ad attuffare il capo in un rinfrescatoio quivi vicino [...].

Finalmente arrivò pur l'avviso, che 'l letto era all'ordine. Entro in camera, et veggo una gran lettieraccia di noce antica, che mi cred'io che fosse già del Duca di Atene, dove sicuramente per le molte ritirate ne' fogliami, ne' capitelli, et nelle cornici \& in mille altri luoghi bisogna, che vi alloggiassero al meno dui millioni di quei animali multiplici notturni di Fidentio. Io dico però a Gaspari, [assistente del nostro] che mi tiri un matarazzo in terra, che quello non mi par punto luogo per poter riposare: risponde egli (e prudentemente) fuggirete i cimici per raccomandarvi a i pulci: all'hora io rimettendomi a lui, dico fratello, mettimi dove vuoi. Egli però sopra la tavola dove hora scrivo, la quale se non è paralitica, fu almeno morsicata dalla Tarantola senza esserne mai guarita, mi raggruzzolò un poco de lettuccio, accostando la tavola al muro per assicurarla; \& nel salirvi sopra poco mancò, che la fabrica non rovinasse; ma perché si fermò poi assai facilmente, io bramoso di dormire mi corcai subito, \& appena fui tra le lenzuola, che mi addormentai. Il servitore nell'andarsene, smemorato, com'egli è sempre, lascia il lume acceso in terra; \& io che non posso dormire con così fatta compagnia, destomi a caso indi a non molto, volendo spegnerlo senza levarmi di letto, mi servij a questa impresa delle 
pianelle, che m'erano vicine sopra una sedia, ma mi fallirono: il guanciale medesimamente non fece colpo; di maniera, che postomi in ginocchi con un poco di colerica risolutione vi lanciai il cappezzale, il quale smorzò bene la candella, ma nel medesimo tempo mi trasse anco seco insieme con tutto il letto, non senza gran pericolo di rompermi il collo, o di stroppiarmi al meno: la tavola non cadde affatto, ma uno de' capi restò appoggiato parte ad un trespolo, e parte ad una sedia: di maniera, che io al buio non sapendo come mi fare, né dove io mi fossi, né sentendo hormai più persone per l'hosteria, mi accomodai nel letto così pendente al meglio che potevo, in modo che la mattina mi vidi in sito tale da non invidiare ad una delle più furiose inventioni di rovine, che facesse mai il nostro Tintoretto. [cc. 3 or-3 Ir]

Davvero admiranda questa chiusa sull'iconicità convulsa del Robusti, ${ }^{\mathrm{I}}$ in cui corpi a testa in giù come precipitanti in prospettive teatralmente audaci si umiliano nella comparazione comica con il povero Gio. Battista penzolante in un risveglio penoso. Tutto naturalmente si svolge in una locanda («sono badialmente all'bosteria»), comunica in apertura l'epistolografo, c. 30r), luogo cruciale per le narrazioni picaresche e avventuroso-realistiche, iper-luogo rappresentativo del Quijote già dai suoi primi capitoli. Naturalmente l'onnivora letterarietà del Leoni attinge a svariate occorrenze cinquecentesche, ove le locande-inferni sono ospitate in scritti burleschi, satirici, grotteschi. Ne fa fede la citazione di Fidenzio che il Leoni esplicita nella sua lettera; il richiamo è all'epico ternario dello Scroffa $\mathrm{O}$ da me celebrando in mille pagine, in cui lo sventurato ludimagistro si ritrova nel letto lurido di una taverna: "Vennermi intanto legioni a invadere / d'animali multiplici et deterrimi, / tal ch'io non credea mai poterne evadere». ${ }^{\text {I9 }}$

Anche la missiva A M. Gio della Gostena, scritta da "Giudemburg» (Judenburg in Stiria, Austria) il I6 ott. I573 rimandava a questo immaginario, con ancor più animazione e trambusto di personaggi rispetto alla solitaria notte disastrosa della lettera al Badoaro. Leoni descrive una solenne ubriacatura in un'osteria, qui con tratti che ancor di più fanno pensare a Cervantes o alla novela picaresca; vale la pena citare il testo quasi per intero:

Arrivammo qui hieri sulle 3 . hore dopo mezo dì, \& con la solita parata di collane d'oro, et di valigie guarnite si facemmo accarezzare dall'hoste alla grande; intanto ch'egli, la moglie, et due figliuole belle come quella nostra 
Giuditta, vennero non pure a riceverci, \& honorarci dell'ordinario Billicom, ${ }^{20} \mathrm{ma}$ intervennero anco alla cena, la quale fu abondante più del solito, perché essendoci noi stimati qualche cosa di più di quello che siamo, \& sapendo noi assai ben conservar il decoro della Corte Cesarea, fummo \& dall'hoste, \& da alcuni cittadini presentati, \& trattati veramente all'imperiale. L'ingresso della cena fu tempestivo \& queto, ma l'uscita molto diversa; si cominciò ne' primordij a scaramucciare con qualche brindes piacevole, \& tra scherzi, risatelle, \& qualche vezzo amoroso, dove bisognava, si conducemmo a un gran conflitto di Corauz, ${ }^{21}$ nel quale il nostro Capitano fece prove inaudite, \& l'hoste ruppe seco a corpo a corpo molte lancie; \& vennero anco di modo alle prese, che l'uno \& l'altro cadde, quegli in braccio della moglie, \& delle figliuole, \& questi sul dorso della medesima mensa. Io sostenendo da un corno la carica feci quanto potei; ma finalmente vedendo che questi dui gran campioni nel corpo della battaglia giacevano, non so se stanchi, o feriti, io volendo pur riconoscer lo stato delle cose, vedevo che le muraglie s'aggiravano, il palco fuggiva all'insù, \& la mensa chi v'era d'intorno tutta si dimenava; $<$ se $>$ stendevo un braccio per prender una salvietta ella fuggiva; se mi rizzavo per voler sottrarmi dal campo, \& marciare alla volta del letto, vedevo più raggi, \& più splendori, che non conduce seco la girandola del Castello in Roma; \& se volevo mover un passo parevami che 'l suolo sfondasse. Di modo che conoscendomi io veramente ubriaco insieme con tutto il rimanente della brigata, \& tanto più che vidi dalla moglie, dalle figliuole, \& da' servitori esser portato l'hoste pesolone alla camera sua, andavo fra me stesso desiderando aiuto, \& non sapevo, \& non potevo chiederlo. Venne pur in tanto il servitore del Capitano per condurlo a dormire, \& in fine dopo qualche fatica destatolo, egli riassonta subito la solita bravura, con gli occhi infocati, con la fronte \& le ciglia inhispidite, \& con la bocca tutta spumante, rizzossi \& gridò ammazza ammazza. Alla qual voce tonante, \& risonante io subito mi riscossi dallo stupore, \& mi risentii tutto; \& attento per quanto potei, andai avvertendo quello ch'egli faceva per poter aiutarmi anch'io. Con una guattatura bieca, e sfavillante fulminando vino e furore contra il povero famulo, disse ehlà dov'è la mia trabacca; a chi dich'io, dov'è la mia tenda? \& in uno stesso tempo si spicca dalla tavola per girsene; ma quasi slitta nel mese di Gennaio, sdrucciolò con tanto impeto che trasse seco non pur il suo massiccio corpaccio, ma il misero servitor ancora, che solo tra tanti di quella casa si trovò per avventura illeso in così fiera battaglia; \& ambidua se n'andarono ad investire in un banco opposito. Io nella medesima ubriacchezza sentendo pur non so che stimolo di pietà, 
mossimi per soccorrerli, ma non potendo reggermi anch'io come bisognava, men'andai a ritrovarli nell'istesso luogo, \& nell'istesso modo, \& avvenne, o fosse il moto, o l'abondanza del ripieno, che gran parte di quello, ch'entrò già per la bocca, se ne uscì ancora per la medesima strada, \& andò per mala sorte a cader sopra la testa del Capitano, che se ne stava boccone su quel banco, il quale colto da quel caldo, \& da quell'umido improviso, grattandosi un tantino la testa, rivolse subito il volto all'insù, forse per vedere di dove gli veniva così fatta pioggia; \& io replicando la carità, non potendo contenermene, gl'impiastrai con mio gran dispiacere tutto il mascarone della medesima vernice; \& così scarico in parte di quello che mi aggravava la testa, \& lo stomaco, carpone carpone mi condussi subito a la camera, \& senza altrimente spogliarmi mi lanciai nel letto. Sentij in tanto, che 'l servitore asciugando, \& forbendo il Capitano al meglio che poteva, fece di gran fatica per condurlo a letto, ch'era nella medesima camera mia; dove arrivato non deposto punto della solita terribilità, disse, apri quella valigia, ch'io vo' dormirvi dentro; \& rispondendo il servitore, eh Signore, che questo è 'l vostro letto, di gratia spogliatevi presto: egli dato di mano a un arcobugio, replicò, se non l'apri tu, l'aprirò ben io, quivi debbo dormir, \& non altrove per sicurtà delle cose mie. Et perché la bocca dell'arcobugio era volta verso il mio letto, con tutto che la valigia gli fosse dirimpetto, \& si correva pericolo di provar forse foco più fastidioso di quello che ardeva nell'una, \& nell'altra fornace, gridai al servitore, \& consigliailo, che in nome di Dio lo lasciasse coricarsi dove voleva; come fece. Pose però il buon Capitano la testa dentro alla sua valigia, \& il rimanente del corpo giacque sopra feltri, tapeti, \& similia, \& così s’addormentammo tutti. [cc. 23r-24v]

I motivi del vomito, dell'ubriachezza, delle bravate sono tutti squisiti segnali di una narratività realistica che fra Cinque e Seicento si diffonde sempre di più, e Leoni, pur nel suo piccolo, contribuisce a questa baraonda che in autori come Alemán, Cervantes o Quevedo, facendosi spesso cupa e pensosa, spianerà la strada al romanzo moderno.

Tornando alle missive con allegati componimenti in versi, soffermiamoci per un poco su quella in cui da Napoli, s sett. Is80, Leoni scrive a Lelio Gabrieli, "Cameriero di N. Signore a Roma» (ruolo che il Leoni avrebbe sperato per sê), inviandogli un sonetto che il destinatario aveva già letto precedentemente a Pisa. "Il sonetto mi venne fatto in età di I7. anni in que' fervori, \& in quel caldo di sangue, che fa la gioventù 
licentiosa ne $i$ suoi concetti, \& istimatrice di se stessa oltre quello, che comporta il dovere» (c. 25r). La Gentildonna di cui si piange concettosamente la morte fu moglie di Francesco Moresino e figliola di Giovanni Soranzo, entrambi nobili veneziani. Leggiamo il sonetto:

Fece Natura già donna sì bella,

Che vedendola Amor senz'alma ancora,

Come alma si pensò di entrarvi a l'hora,

E star poi sempre, \& habitar con ella.

Hor mentre che del Mondo e questa e quella

Parte sì dolcemente arde e innamora,

Venere il cerca, \& ne dimanda ogni hora,

Né pur trova di lui segno, o novella.

Onde confusa, dolorosa, e mesta

Piangendo il chiama, \& ei tosto che l'ode,

Esce per consolarla, ahi cruda sorte,

Et Cecilia senza alma se ne resta,

Che tal fu il nome: onde hor si lagna, e rode

In van Natura, il Mondo, Amor e Morte.

[c. 26r]

Variante funebre sul topos dell'anima trasmigrante, o meglio di Amore stesso che si insedia nell'anima della Donna, il sonetto contamina questo immaginario con il celebre mitema di Venere alla ricerca di Amore, oggetto di canto dell'idillio di Mosco, poi ripreso e rielaborato nel prologo e nel cosiddetto epilogo Amor fuggitivo dell'Aminta tassiana. ${ }^{22}$

Nota - e notevolissima - è una missiva al pittore Francesco Montemezzano, ${ }^{23}$ in cui da Venezia il Leoni invita l'artista a Roma il 6 agosto '89. La celebrazione della grandezza pittorica dell'ambiente romano viene richiamata anche da un ricordo dell'epistolografo, coinvolgente un veneziano di primissimo ordine come il Vecellio:

Et io mi ricordo haver sentito dire a Messer Titiano, mentre che nella mia pueritia, per apprender anco qualche cosa di pittura, me n'andavo alle volte in casa sua, che dopo esser egli stato in Roma, haveva grandemente migliorato le cose sue. [c. $28 \mathrm{v}$ ]

Con un viatico più autorevole di così.. I pittori d'oggi, aggiunge Leoni, escludendo ovviamente maestri sublimi come Tintoretto o il Veronese 
(del quale ultimo il Montemezzano era probabilmente allievo) e il Montemezzano stesso, errano nel non seguire le morbidezze, la naturalità, il realismo degli affetti che mostrano gli artisti in Roma, talché

vedemo spesso in una battaglia figure con mostacci ridenti, come se giocassero a Tarocchi; \& altri in uno sposalizio così ingrugnati, come se fossero per fare alle pugna. [c. 29r]

Naturalmente, si immagina Leoni, l'interlocutore risponderà sghignazzando e sostenendo che non mancano maestri li dove l'artista risiede, e allora

nel qual caso io vi risponderò, galant'huomo mio senza colera, con questa occasione di invitarvi a Roma ho voluto passare in così fatto discorso, \& ve ne ho fatto uno schizzo così di carbone, $\&$ di gesso; se vi piace, servitevene, se non, fatene uno scartoccio da orpimento, \& da terra d'ombra, \& non bravate. [cc. 29r-v]

Il linguaggio è ancora una volta vivacissimo e degno di un ottimo scrittore di epistole; in particolare quel riferimento ai pittori maldestri che rendono caricaturali e contraddittori personaggi di quadri di battaglia o di sponsali fa pensare all'evoluzione "realistica", con influssi nordici, che si produce nella pittura italiana, soprattutto lombarda ma non solo, tra fine 'soo e inizi '600. D'altronde lo stile del Leoni drammaturgo è tutto ripieno di succhi esuberanti che tendono all'esaggerazione immediatamente prossima cioè barocca. Infatti un altro luogo leoniano in cui presentiamo certe ossessioni care alla lirica che chiamiamo "marinista" ̀̀ dato dalla lettera al cardinal Gambara, a Bagnaia, da Romas sett. Is 8 I (cc. IOv-IIV). Qui l'autore descrive le acque cadenti del luogo ove si trova il destinatario e propone una invenzione capricciosissima per filtrare l'acqua e trasformarla in «spilli» sgocciolanti simili a spighe di grano. Prolegomeni al descrittivismo secentista di fontane e giochi d'acqua ingegnosi, pure con una razionalità ingegneristica che non sarà estranea anch'essa al gusto a venire (si pensi solo all'Imperiali).

Un altro riferimento al pittore Montemezzano è presente in una lettera a Girolamo Zeno scritta da Velletri il 4 novembre I585. ${ }^{24}$ La missiva è ancora di quelle giocose, con uno stile comico assai dovizioso sul piano lessicale e mosso nel procedimento narrativo. Vi si narra di un viaggio da Marino a Velletri, in cui protagonista è «un Aiutante di Camera 
dell'Arcivescovo mio signore» (ovvero l'arcivescovo di Napoli Annibale di Capua), descritto in modo più che gustoso:

Napolitano di Patria \& di humore, hebbe in Roma per sua cavalcatura nel presente viaggio una Mula da vettura di passo assai commodo, ma però bizzarra secondo il costume della specie. Questi sedendovi sopra pareva un Astolfo descendente dal cerchio della Luna; con un gran paio di bracconi gonfi per molta bambace riempieva maestevolmente la sella: poi co 'l feltro attorno, cappello d'ormisino, \& stivalletti incerati (livrea assai capricciosa in questi tempi) cavalcava pavoneggiandosi. [c. I 8v]

Il simpatico personaggio si dimostra assai faceto durante il percorso, senonchéper un incidente imprevisto (il suo cappello gli viene portato via da un ramo d'albero) è costretto a scendere dalla sua cavalcatura. Tentando di riprendere il controllo della mula non vi riesce, ed è costretto a inseguirla ansando, mentre il Leoni lo accompagna nascondendo un riso prorompente. «Mula bene mio ferma, ferma bene mio» supplica grottescamente il povero aiutante di camera. Alla fine la mula si arresta, trovandosi a suo agio in un campo fra altri somari, altrimenti il povero disgraziato «bamboccione» sarebbe entrato poco trionfalmente a Velletri preceduto dalla sua cavalcatura, appiedato e sfatto. Conclusione:

Non ho potuto contenermi di non mandarvene così subito questo ritratto al meglio, ch'io ho potuto di chiaro e scuro, il quale non doverà forse dispiacere al nostro Montemezano, essendovi alcune pennellate imitanti la sua maniera. [c. I $9 \mathrm{v}$ ]

Perché il racconto verbale di Leoni dovrebbe risultare gradito all' amico Montemezzano, anzi addirittura sintonico con «la sua maniera»? Può trattarsi solo di un gioco tra sodali, burlesco se non antifrastico? Del pittore conosciamo poche opere superstiti; se osserviamo una delle più celebri, la Presa d'Acri della Sala dello Scrutinio di Palazzo Ducale, verifichiamo un gusto per il movimento, l'impeto, la concitazione dei corpi e le loro vibranti muscolature, la numerosità delle figure, lo scorcio ardito. C'è qui qualcosa che può farci sospettare che Leoni si riferisca propriamente alla maniera del Montemezzano? Non saprei rispondere, francamente. Quel tanto di splendidamente confuso e mescolato nell'opera al Palazzo Ducale mostra un vago parallelismo col gusto mescidato ed eteroclito del Leoni drammaturgo e scrittore. Non a caso della Presa d'Acri di Montemezzano scriverà Zanotto nel $1860:{ }^{25}$ "Imperocché, 


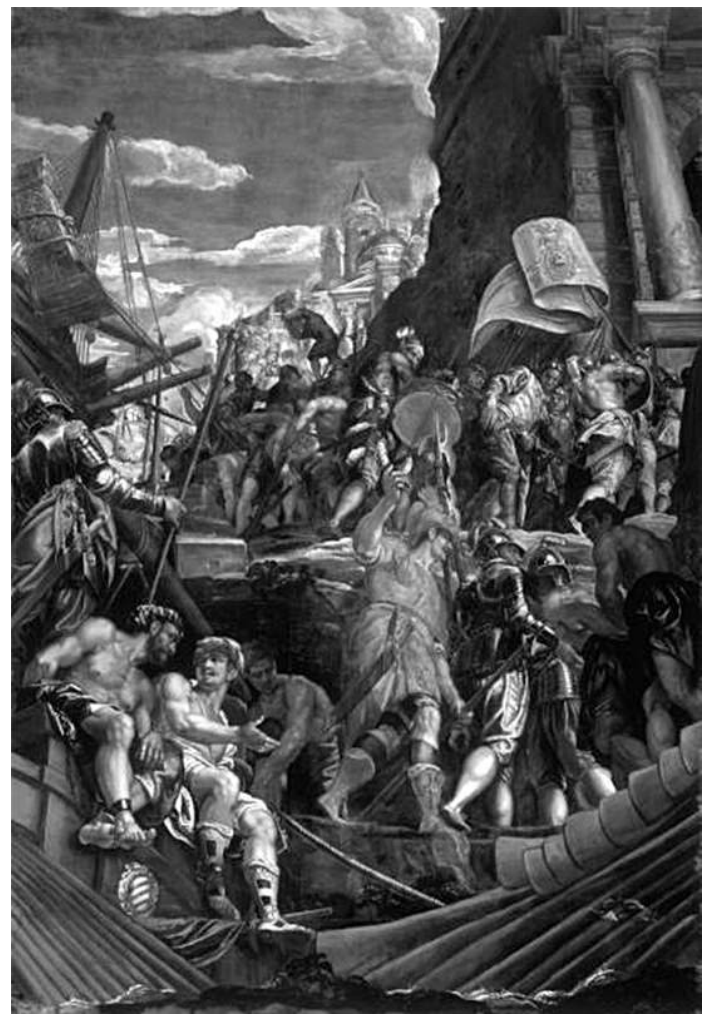

Fig. I : Francesco Montemezzano Presa d'Acri, Palazzo Ducale, Sala dello Scrutinio, Venezia.

non presenta essa ottima composizione; non, come vedemmo, unità di soggetto; non disegno regolato, né proporzione di forme; non espressione che consuoni ai varii affetti che mostrare deveansì. Critiche a un anticlassicismo sull'orlo del barocco che risuoneranno nei secoli seguenti e che potrebbero congiungere le sorti, in questo scorcio cinquecentesco, degli amici Leoni e Montemezzano.

Certo se l'animosità (pur capricciosa e comica) del racconto leoniano può far pensare alla veemenza del Montemezzano, il richiamo al chiaroscuro può evocare certe opere sacre del pittore, fra cui una Pietà recentemente a lui attribuita, conservata alla St John the Baptist's Church a Tunstall, nel Lancashire.

Chiaroscuro e pennellate in un'atmosfera tra Tiziano e Tintoretto, e ci perdonino gli storici dell'arte per la semplificazione. E questa la maniera cui si riferisce Leoni, anche a prescindere dall'eventuale ironia in chiusa di epistola? Forse. Certo è che il pittore doveva essere in 


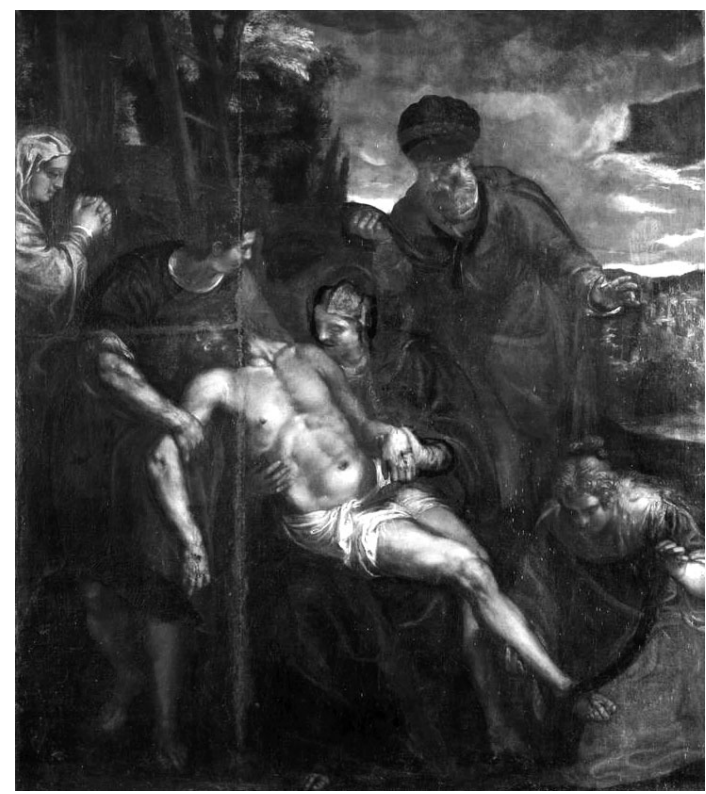

Fig. 2. : Id. (attr.), Pietà, St John the Baptist's Church, Tunstall, Lancashire.

armonia con lo spirito humoroso della cerchia del Contarini (cfr. infra); di lui scrive il Ridolfi:

Hebbe gratia nelle conversatione, \& abbondò di molti amici, mediante a' quali otteneva tutto quello che bramava, \& impiegava volontieri l'offitio suo nell'altrui servigio. Ma datosi soverchiamente Francesco a' piaceri amorosi, invaghitosi d'alto oggetto, provò anco più fiera la caduta terminando la vita (per quello che si dice) di veleno circa l'anno i60o, nella sua più fiorita età. ${ }^{26}$

Nella Parte terza ${ }^{27}$ delle lettere del nostro abbiamo ancora rilevanti missive su cui soffermarci. Il Lodovici firma la lettera di dedica del volume «Alli Clarissimi Signori del Mezzato Contarino ${ }^{28}$ trasferito nelle stanze del Clarissimo Signor Giacopo Marcello». Si definisce "discepolo» e quasi figlio del Leoni, che lo ha costituito «possessore, et berede delle sue scritture». ${ }^{29}$ Seguono, nell'edizione I596, carmi latini in onore del Leoni di Ulisse Colloredo, di Incerto e del Lodovici stesso. Poi la tavola delle lettere scritte dal Leoni a suo nome, indi di quelle vergate come segretario del card. di Lenoncourt. Di queste ultime le uniche datate segnano I587 e I588. Tra le altre, sono indirizzate al Lenoncourt 
lettere fra il luglio '88 e l'agosto '8g. Il paratesto di soglia dell'edizione I600 è ovviamente comprensivo di tutte e tre le parti delle lettere.

Le doti di narratore del Leoni si ritrovano in più di una missiva di questa terza parte. Pensiamo a quella indirizzata a un cardinale non nominato sull'esecuzione della Regina di Scozia (del I4 marzo I587). ${ }^{30}$ Molto intensa e patetica, fra novella elegiaca e dramma storico, la narrazione della decapitazione, con particolari realistici ed evidentes quali il sg.: "fu dal Carnefice per tutte le fenestre della sala mostrata la testa alla moltitudine del popolo basso, che era ridotto nel cortile». Su questi aspetti di espressivismo storiografico, topici di un narratore enargico di cose veramente accadute, segnaliamo anche la missiva da Roma del I585 a Giorgio Contarini, su un tumulto napoletano con atroce linciaggio. ${ }^{3 \mathrm{I}}$ Esempi ecfrastici ben piu leggiadri sono ugualmente motivati da esigenza di ipotiposi anche puntigliosa; al vicinissimo Lodovici invia fra l'altro una ricca missiva sul carnevale di Venezia (25 feb. I596), ${ }^{32}$ con un virtuosismo descrittivo particolarmente dettagliato nella raffigurazione degli abiti, oltre che delle altre squisite occasioni di divertimento. Nella lettera al medesimo del 25 gennaio inviava invece un sonetto sulla guerra d'Ungheria, Questa d'anni e di fede Aquila bianca, che non merita una citazione integrale.

Ad Alessandro d'Este manda due madrigali l'8 sett. I595, ${ }^{33}$ senza riportare $i$ componimenti nella stampa. Sempre in ambito di lettere accompagnatorie di testi lirici, ricordiamo che a Girolamo Zeno scrive una lettera da Napoli il 20 luglio I584, ${ }^{34}$ dove il soffermarsi a contemplare le rovine di Pozzuoli gli ha fatto risorgere nello spirito e nella mente «concetti passati», e quindi l'impulso a poetare, nell'occasione presente. Si notino i lemmi concetto $e$ occasione, determinanti per la vicenda lirica fine Cinque-primo Seicento. Riportiamo il sonetto:

Eterni fuochi, \& voi sacri bollori, Che altrui giovando in minacciosa vista, Serbate quello, onde natura acquista Novella fede a i suoi stupendi honori;

Occolto incendio d'insueti ardori, Che in ampla cava horribilmente trista Con un atro palor, che l'alma attrista, Te stesso mormorando apri e svapori;

La fiamma, che nel petto Amor mi accese (Lasso), fatta vorace e sempiterna, 
Vostro aspetto crudel (misero) adegua.

Qui gode Amor del foco suo l'imprese,

E 'l cor, che sospirando si dilegua,

Consola altrui nella mia pena eterna. ${ }^{35}$

Tipico sonetto di contesto "fiammeggiante" napoletano e meridionale (manierista?), con la consueta contrapposizione fra il fuoco naturale $e$ quello amoroso; nulla insomma di straordinario nell'escogitazione ingegnosa come invece capita in certi madrigali.

Spostandoci nella sfera della produzione teatrale del Leoni, anzi della sua riflessione meta-teatrale ospitata dall'epistolario, segnaliamo intanto la lettera non datata da Venezia a Giovanni de' Medici, in cui spedisce l'Antiloco, ovvero la sua "tragicomedia». ${ }^{36}$ Ma sull'Antiloco $e$ in difesa dell'istituto tragicomico si veda l'importante missiva a Giorgio Contarini da Venezia. ${ }^{37}$ Innanzi tutto il Leoni rivendica, potremmo dire, il valore di "realismo" e di "rispecchiamento" del vero da parte del teatro tragicomico: l'alto e il basso si mescolano nella effettiva realtà, ad esempio negli infiniti intrighi che la complicano:

poiché si danno in natura accidenti, che risultano da amori et da negotij gravi, \& comporta l'uso delle Corti, che persone basse proportionate alle Comedie maneggino tra grandi materie gravissime, et ammettere la Tragicomedia come misto non repugnante al vero \& al possibile [...]; dico che sì come intromettendo io una persona Comica tra le persone Tragiche, non levo a loro la propria gravità, né meno altero la conditione di quell'altra, ma dalle attioni proprie de gli uni, \& de gli altri faccia nascere il misto dell' accidente. ${ }^{38}$

Ho messo in italico l'ultima espressione, perché mi pare contenga una indicazione straordinariamente vivace e sintonica col teatro europeo del momento e degli anni subito a venire. La teorizzazione della tragicommedia pastorale di Guarini è qui in secondo piano, assolutamente. Il discorso del Leoni può valere per Shakespeare o per Lope, per drammi di ambientazione storica, cupi e infiltrati dal grottesco e dal servile talora infame. E poi è la parola accidente a travolgerci: Leoni è dalla parte di coloro che difendono il tragico e il comico mescolati perché cosi vuole la natura (Lope docet), ${ }^{39}$ anzi la natura fattuale. Nonostante l'Antiloco certo sia lontano da sperimentazioni europee cosi apicali, tuttavia Leoni, che difende la libertà di comporre forme moderne (come 
del resto, egli soggiunge, fanno Arte, Musica, Architettura, Scienza), sposta la sua tragicommedia dalle selve alla città (Corinto), ponendosi su una linea forte che dal Giraldi Cinzio arriva al pieno teatro secentesco. Altre interessanti innovazioni rivendicate sono ad esempio l'eliminazione dei monologhi, in virtù di una scioltezza drammatica fluida, l'esclusione dei Cori, sentiti evidentemente come troppo classicistici, la semplificazione della vicenda, l'abolizione del canto, il tacitare noiosi interlocutori quando spezzano la tensione della scena. Tutte proposte che, anche quando restano isolate, non mancano di una consapevolezza epocale davvero lucida. Il Leoni non manca poi di concepire l'apparato scenico con competenza e correttezza professionale. Lo verifichiamo in una missiva indirizzata ancora al Lodovici, tutta concentrata sulla scenografia della Falsa riputazione, ${ }^{40}$ da Ferrara 20 feb. I596. Qui egli sottolinea la necessità di un décor "astratto", consono alla natura intimamente allegorica della pièce. La scena sarà quindi "principalmente Ideale, si come le persone sono parimenti ideali》. Ma naturalmente si vedranno case, strade ecc., sicché il luogo «che si concede loro in astratto, sia lecito ancora, \& si faccia visibile \& tolerabile in concreto». D'altronde, non si rappresentano forse quadri scenici «ideali» - ma visibili! - quando si inscenano gli dèi dell'Olimpo nelle loro stanze? Particolarmente interessante dunque questa nuova accentuazione su una concezione "ideale" e simbolica di un teatro che rappresenta un plot allegorico morale, secondo un genere praticamente quasi nuovo ( $v d$. supra), a testimonianza ancora della vis sperimentale del nostro.

Roberto Gigliucci 
I. Vd. ora D. E. Rhodes, Giovanni Battista Leoni diplomatico e poligrafo. Appunti bibliografici, bibliografia degli scritti, regesto della corrispondenza, premessa di P. Procaccioli, Manziana (Roma), Vecchiarelli, 2013.

2. Recitata in Venetia, l'anno M.D.XCV. da gli Academici Pazzi Amorosi, In Venetia, G. Battista Ciotti, I 595: se ne ebbero ristampe e ulteriori edizioni almeno fino al I620. Per ogni dato bibliografico il riferimento implicito è a Rhodes, Giovanni Battista Leoni, cit. Vd. anche in particolare F. Bertini, In traccia del cavalleresco nella «Roselmina» di Giovan Battista Leoni, in «Schifanoia», 34-35 (2008), pp. I 57-64.

3. Vd. di chi scrive Tragicomico e melodramma. Studi secenteschi, Milano, Mimesis, 20 I I, p. 9 con bibliografia in nota, cui aggiungere il classico volume di L. G. Clubb, Italian Drama in Shakespeare's Time, New Haven and London, Yale Univ. Press, I 989 , pp. I 8 I -84 .

4. Ovvero l'elemento "satirico".

5. Parte catorze de las Comedias de Lope de Vega Carpio [...], Madrid, J. de la Cuesta, I620, c. $219 \mathrm{~V}$.

6. Vd. G. Benzi, Il «Parthenio» di Francesco Pona, in «Filologia e critica», in. c. s.

7. Sulle varianti possibili della tragicommedia mi permetto di rimandare al mio Tragicomico (Napoli, Guida, 2013) quantomeno per la bibliografia ivi presente.

8. Data questa produzione teatrale fortemente ibrida e consapevolmente eteroclita, risulta singolare che Leoni sia anche l'autore di un Parere negativo e "regolistico" sulla tragicommedia Il Pastor Costante di Cataldo Antonio Mannarino (i606), parere cui il Mannarino rispose con una Apologia nel i608: la questione andrà approfondita. Vd. M. Leone, Fenomenologia barocco-letteraria. Saggi, Galatina, Congedo, 201 2, pp. I 93 sgg, e i saggi di Grazia Distaso, ivi cit. in nota.

9. Cfr. M. Moretti, La celebrazione dei Della Rovere in due dipinti di Giorgio Picchi, in I Della Rovere nell'Italia delle corti, vol. II, Luoghi e opere d'arte, a cura di B. Cleri, S. Eiche, J. E. Law, F. Paoli, Urbino, QuattroVenti, 2002, pp. I4I-66, p. I4I n. 2.

ı. Come ci indica una informata nota di Emilio Russo (Studi su Tasso e Marino, Padova, Antenore, 2005, p. 72 n. I6).

i i. Emanuele Antonio Cicogna, Delle iscrizioni veneziane, vol. IV, Venezia, G. Picotti, I834, pp. 649 sg., offre alcuni dati biografici: trascrive una lettera al cardinal Pinello in cui forse Leoni stesso, che dice di essere stato al servizio del Commendone, si rammarica negando di essere autore di una certa pubblicazione contro la Santa Sede, forse proprio i Due discorsi: la missiva è all'Oliveriana di Pesaro, T. XIII Monumenti Rovereschi mss. p. I 29. Cicogna poi ricorda che Leoni fu carcerato per ordine del Consiglio dei Dieci il is nov. i60o, ma poi subito rilasciato perché riconosciuto innocente. Non sa però perché. Rhodes (Giovanni Battista Leoni, cit., p. 73) indica ipoteticamente Francoforte come luogo di edizione dei Due discorsi.

I 2. Rhodes, Giovanni Battista Leoni, cit., p. 7 I indica un secondo esemplare alla Nazionale di Firenze «restaurato dopo l'alluvione del ig66». 
I3. Occasioni nella lirica manierista, in Festina lente. Il tempo della scrittura nella letteratura del Cinquecento, a cura di C. Cassiani e M. C. Figorilli, intr. di N. Ordine, Roma, Edizioni di Storia e Letteratura, 20I4, pp. 99-100. Chiedo venia se talora ripeto qui alcuni dati desumibili da questo saggio. D'altronde, a quanto mi consta, sul Leoni non c'è neppure una voce del Dižionario biografico degli Italiani Treccani.

I4. Personaggio notissimo della Roma di fine 'soo, attivo nell'Accademia dei Tiberini e coinvolto in prima persona nella elaborazione del Rogo amoroso o Rogo di Corinna di Torquato Tasso (cfr. il mio Giù verso l'alto. Luoghi e dintorni tassiani, Manziana, Vecchiarelli, 2004, pp. 53-7I).

I 5 . Il Leoni è a Parigi dal 3 I gennaio al 20 settembre I 587 : cfr. Rhodes, Giovanni Battista Leoni, cit., pp. 24-25.

I6. Citiamo dall'edizione dei Consigli de gli Animali, Venezia, G. Griffio, i 552 , c. $26 \mathrm{r}-\mathrm{v}$.

17. Delle lettere familiari di Gio. Battista Leoni, parte seconda [...], Venezia, G. B. Ciotti, 1593 .

I8. Nel 30 sett. '98 a Don Alessandro d'Este il Leoni invia un'orazione in memoria del Tintoretto recitata in Accademia 20 giorni prima: «compositione capricciosa appunto come sono state le opere di esso Tintoretto» (Terza parte I 5 r-I 5 V, p. I 5 r). Purtroppo il testo non è presente. Leoni difende la molteplicità di stili usati, in conformità alle molte materie.

19. Vd. Camillo Scroffa, I cantici di Fidenz̧io con appendice di poeti fidenziani, a cura di P. Trifone, Roma, Salerno Editrice, I98 I, p. 35, XIX, vv. I 2 I-23.

20. Storpiatura per willkommen.

2 I. Forse riferimento alla battaglia di Coutras del i 587 ?

22. Vd. ora Torquato Tasso, Aminta, a cura di M. Corradini, intr. di G. Baldassarri, Milano, Rizzoli, 201 5, pp. 59, 223.

23. Vd. voce del Dizionario biografico degli Italiani di Giorgio Tagliaferro e soprattutto Id., Quattro Jacopo per Montemezzano, in «Venezia Cinquecento», Roma, Bulzoni, XI, 200 I, 21, pp. I 45-54.

24. Segnalata da Tagliaferro, ivi p. I 43.

25. F. Zanotto, Palazzo Ducale [...] Volume Terzo, Venezia, G. Antonelli, i860, tavola CLXXX, p. 8.

26. Carlo Ridolfi, Delle maraviglie dell'arte [...] Parte Seconda, Venezia, G. B. Sgava, I648, p. I 37 .

27. Abbiamo tenuto presente le due sgg. edizioni: Delle lettere del Signor Gio. Battista Leoni. Parte terza. Nella quale si contengono negotij, avvisi, et complimenti gravissimi. Raccolte et pubblicate da Vincenzo Lodovici. [...] In Venetia, M.D.XCVI. Appresso Gio. 
Battista Ciotti Senese, al segno della Minerva [esemplare: Alessandrina, K.d.33. $\mathrm{f}^{2}$; Delle lettere familiari di Gio. Battista Leoni. Divise in tre parti, nelle quali ce ne sono molte di Negotij Avvisi, \& Complimenti gravissimi [...], In Venetia, G. B. Ciotti, I600 [esemplare: Casanatense K.VIII.I 9]. Citiamo da entrambe excerpta testualmente coincidenti.

28. Il «mezzato Contarini» era «un circolo che come molti altri si riuniva nelle stanze del mezzanino in un palazzo che nella circostanza risulta essere quello del senatore Jacopo Contarini» (Tagliaferro, Quattro Jacopo per Montemezzano, cit., p. I43). Il club Contarini era probabilmente assai vicino all'Accademia Veneziana Seconda, inaugurata nel I 593 , che ebbe Leoni tra i fondatori; cfr. ivi, pp. I42, I 50 , I 2 n. 3. Vd. anche Rhodes, Giovanni Battista Leoni, cit., pp. 3 I sg.

29. «Insomma, una specie di esecutore letterario?» si domanda il Rhodes, ivi, p. 32 .

30. Cc. $6 r-8$ r ed. I $596=209 \mathrm{~V}-2 \mathrm{I} 2 \mathrm{r}$ ed. $\mathrm{I} 600$.

31. Cc. I7v-igr ed. I 596.

32. Cc. 238 r-239v ed. 1600

33. Cc. I 4 V-I $5 \mathrm{r}$ ed. I 596.

34. C. $76 \mathrm{r} \mathrm{ed.} \mathrm{I60o.}$

35. C. $76 \mathrm{v}$ ed. I 600.

36. Cc. I 3 r-v ed. I $596=2$ I 8v ed. I600. A Ciotti da Rovigo, 4 luglio I 595 , Leoni comunica che il vescovo di Valenza ha tradotto in francese l'Antiloco $(25 \mathrm{v}-26 \mathrm{r}=$ $235 \mathrm{v}$ ).

37. Sempre nella terza parte delle Lettere, cc. $225 \mathrm{~V}-227 \mathrm{~V} \mathrm{ed.} \mathrm{I600.}$

38. Ivi, cc. $225 \mathrm{v}-226 \mathrm{r}$.

39. Lope De Vega, Nuova arte di far commedie in questi tempi, a cura di M. G. Profeti, Liguori, Napoli i999, pp. 58-60.

40. Cc. 223 r-v ed. I600. 